\title{
Visualising the Invisible: Harnessing Augmented Reality and Virtual Reality as Persuasive Technologies for Energy Feedback
}

\author{
Alexander David Fredericks \\ School of Computing and Mathematics \\ Keele University \\ Keele, United Kingdom \\ a.d.fredericks@keele.ac.uk
}

\author{
Zhong Fan \\ School of Computing and Mathematics \\ Keele University \\ Keele, United Kingdom \\ z.fan@keele.ac.uk
}

\author{
Sandra Woolley \\ School of Computing and Mathematics \\ Keele University \\ Keele, United Kingdom \\ s.i.woolley@keele.ac.uk
}

\begin{abstract}
In the last fifteen years, the outlook for engaging direct energy feedback as a method of effectively curtailing domestic energy consumption by at least ten percent has grown more pessimistic as subsequent studies and reviews suggest the impact of such techniques on consumers is much less than hoped. Persuasive technology research has demonstrated multiple successes in promoting resource conserving behaviours however, including in energy consumption related topics. One of the main obstacles with energy feedback is that making use of electricity is an 'invisible' process and doesn't produce any visible feedback to the consumer. This paper suggests harnessing Augmented Reality and Virtual Reality as persuasive technologies to visualise the 'invisible' consumption of domestic activities and promote energy conserving behaviors. A literature section provides short reviews of relevant topics, providing the justification and motivation for conducting this research while an overview of a methodology focusing on a usercentred approach discusses how to incorporate effective consumer feedback into the development process of appropriate energy feedback visualisations. Initial implementation methods for the visualisations are discussed and future work building upon this paper is outlined.
\end{abstract}

Keywords-Energy Feedback, Augmented and Virtual Reality, Persuasive Technology, Smart Meter, In-Home Display

\section{I.INTRODUCTION}

Approximately one third of the UK's electrical energy consumption is from the domestic sector [1], which equates to approximately $17 \%$ of the UK's 370 million tonnes of yearly carbon dioxide emissions [2]. Since 2013, over 13 million smart and advanced meters have been installed in households and SMEs (Small to Medium Enterprises) across the UK [3] as part of the Smart Meter Rollout Programme, with the goal of installing meters in 50 million locations by 2020 [4]. The intention of the Smart Meter Rollout is to curtail energy consumption by providing consumers with feedback on their energy use, allowing them to make informed decisions about their energy consumption. The effectiveness of smart meters and IHDs (In-Home Displays) is highly disputed however.

Persuasive technologies have employed stylised visualisations to influence behaviours and promote certain actions, including those related to energy activities [5]. By making the normally 'invisible' use of energy visible, consumers can be given new motivation for reducing their usage and get direct visual feedback on their consumption. This paper aims to make use of AR (Augmented Reality) and VR (Virtual Reality) as persuasive technologies for displaying energy consumption as stylised visualisations to promote reducing energy use.

The intention of this paper is to act as a position paper for this Work-In-Progress, highlighting the motivation for the

Funded in part by both the European Regional Development Fund and UK Department for Business, Energy and Industrial Strategy. research in focusing on AR and VR as persuasive technologies to address concerns with energy feedback and poor user engagement with feedback in recent years. This paper contributes a literature review of the relevant related research, highlighting the background and justification for this work. A user focused methodological approach and basic implementation of the visualisations are discussed to demonstrate how future research will build upon this to address the issues highlighted in the literature. This is followed by section 2, an overview of relevant Literature with short reviews of Energy Feedback, Persuasive Technologies, and Augmented Reality and Virtual Reality. Section 3, presenting Methodology employed for this research, covering User-Centred Design and the Smart Energy Network Demonstrator. Section 4 discussing the Implementation for the initial development builds used in the research. Section 5 finishes the paper with the Conclusion and suggestions for future work.

\section{LITERATURE REVIEW}

\section{A. Energy Feedback}

A highly cited UK Department for Environment, Food and Rural Affairs review [6] suggests that direct energy feedback may result in consumer reductions of energy use between 10$15 \%$, however later studies were less optimistic with smart meters resulting in ranges below $10 \%$ [7] and from 5\% to $15 \%$ [8]. These lower estimates were reinforced by a meta-study [9] suggesting that although the average across many papers was an $8 \%$ reduction of consumption through smart meter engagement, once taking into account the demographics of the participants and time of year for the investigations, the average energy use reduction could be as low as $2 \%$. Newer studies seem to confirm these lower estimates with papers researching the persistent reduction in consumer energy use from engaging with smart meters at $5 \%$ or less $[10,11]$. The changes in reported ranges of consumption reduction from direct energy feedback are illustrated in Fig. 1, which highlights the relatively downward trend from feedback studies in the last thirteen years. Fig. 1 also demonstrates the wide range of average energy conservation within each study, reflecting the varied response to feedback from varied participant demographics and individual circumstances.

The general consensus from the literature appears to be that simply presenting energy feedback to users is not enough to promote behaviours that result in consistent and long-term reductions in energy consumption. Part of this is due to consumer understanding surrounding energy use. Smart meters and IHDs normally show energy consumption in aggregate, displaying total energy use as a single figure for a household. Consumers struggle to accurately judge how much electricity their appliances consume while functioning 


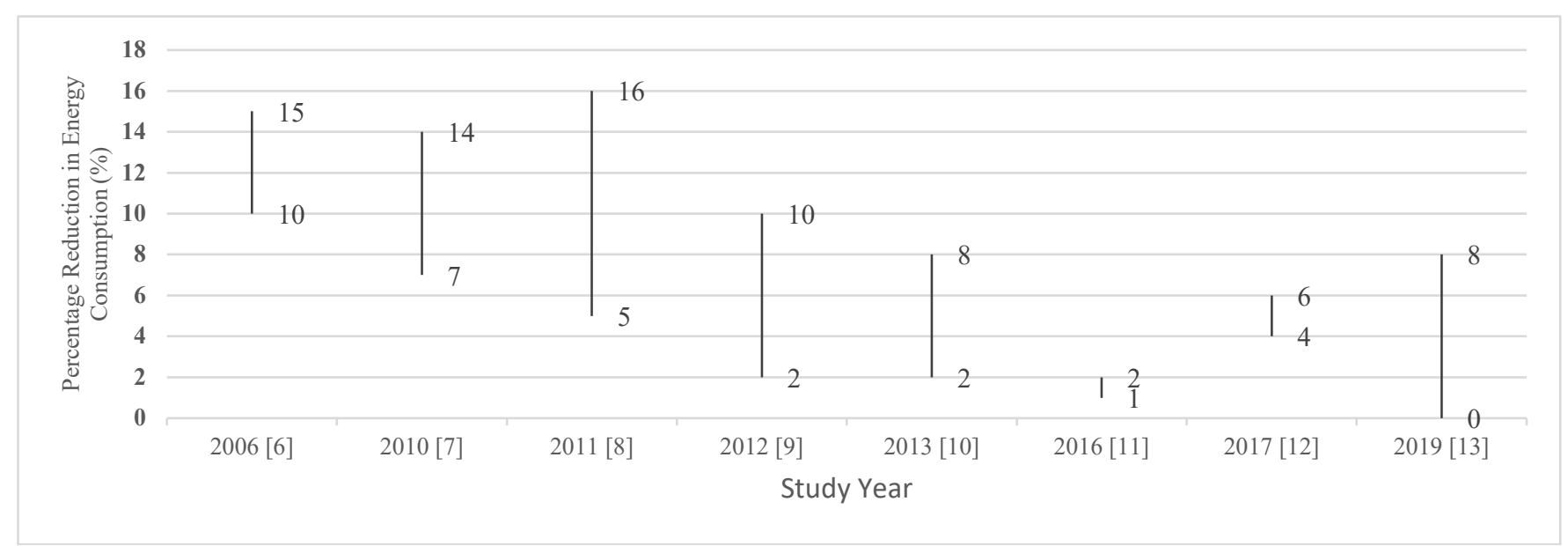

Figure 1. Range of reported percentage reductions in direct energy feedback studies ordered chronicologically by study year.

however [12], making it difficult to identify which behaviours result in high energy consumption when making their decisions from a total use amount. The metrics and units used for displaying energy consumption also influence how consumers perceive their energy use [13] as IHD users develop heuristic methods for understanding the information given by their device [14], they do not necessarily understand what a given metric or unit means and the most sought after means of display may result in the lowest average reduction for consumers [15].

Smart meters and IHDs are likely to simply reinforce current consumer behaviours [16], the devices allow users to identify what their average 'baseline' energy use is, and users aim to stick to that level without reducing their consumptions any further. These devices are reliant on consumers using the provided information to make conscious and informed decisions to reduce their energy use, consuming resources is generally not a conscious decision however and is the byproduct of habitual device or appliance use [17]. Although these meters and IHDs arguably create some level of visibility for the effect of energy consuming behaviours [14], it is generally limited to an aggregate figure or graph that is unlikely to provoke an emotive or memorable response. Making use of more visual stimuli can promote a greater response from consumers [18]. As such, this research looks at developments in Persuasive Technologies for providing energy feedback to prompt energy saving behaviours.

\section{B. Persuasive Technologies}

Although research within Persuasive Technologies has had a growing focus on Gamification to promote behaviour change [5], techniques employing stylised visualisations have traditionally been used.

One of the earliest examples of harnessing Persuasive Technologies to promote energy conservation behaviours used a simple light up device to highlight when the temperature outside was cooler than inside, prompting users to open a window rather than using air conditioning [19]. Similar ambient light displays have been used for energy related behaviours since, such as to highlight to consumers when their available electrical supply is from a renewable source or not [20]. While the use of ambient light displays is a popular method for persuasive visualizations, another technique is to use digital avatars [21]. These avatars portray specific emotions in response to user actions, such as displaying a happy animation when users make energy conscious decisions. Finally, stylised visualisations can be used to display emotive animations that directly reflect user behaviours, such as a virtual display of a natural environment that thrives when conserving energy but dies as the user wastes energy [22].

Whereas ambient lights are used to directly communicate information to users, avatars and stylised displays attempt to directly associate user behaviours to an emotional response to prompt changes in behaviour. The aim of this research is to develop visualisations in VR and AR that harness both of these aspects, using features such as colour, scale and transparency to convey information while the design and style of the visualisations promote specific relevant behaviour.

\section{Augmented Reality and Virtual Reality}

The widespread availability of high-performance mobile devices has resulted in a steady increase of the popularity, accessibility and potential applications of AR and VR [23]. While both AR and VR are used for displaying interactive real time visualisations of $3 \mathrm{D}$ models, VR presents virtual environments to the user while AR superimposes computer graphics over real world scenes captured through a camera.

AR and VR research crosses a multitude of disciplines, with applications for the technologies being suggested across medicine, industry, engineering, education, maintenance and gaming $[23,24]$. From presenting real world simulations, visualising medical procedures directly onto patients and demonstrating molecular structures to interacting with game pieces, highlighting equipment states and displaying graphs and data, AR and VR based solutions have been applied to numerous and varied research problems. The majority of these implementations are scientific visualisations however, representations of real-world phenomena. Visualising data in this manner has received far less attention [25], especially for energy feedback, where there is little investigation into harnessing AR and VR. Although Building Information Modelling has seen some research into this area [26], the visualisations have generally been in tabular and graph form, meaning none of the extra features of AR and VR compared to a normal screen were employed.

By using AR and VR to visualise energy data, this research aims to develop 3D visualisations that involve a greater level of interaction than current methods of energy feedback, while making use of the persuasive methods discussed previously. Employing $\mathrm{AR}$ and VR for this purpose presents an 
opportunity to communicate energy feedback to consumers in a more engaging and visually interesting manner. These visualisations will be based upon user feedback, which will be gathered at every stage of development by following a UCD (User-Centred Design) methodology.

\section{METHODOLOGY}

\section{A. User-Centred Design}

Energy feedback needs to be created with input from consumers [6], this is necessary to ensure the feedback is understandable, provides relevant and usable information, and especially in the case of persuasive visualisations, so that the feedback is effective at appealing to and prompting behaviour in consumers. The need for working with consumers to design feedback can be seen in Fig 1. from the varied ranges of consumption reduction within studies due to differing participant circumstances, responses, and attitudes to direct energy feedback. To facilitate the development of these visualisations alongside consumer feedback, a UCD methodology will be employed so that users (consumers in this case) have input at every step of the development process.

Initial participant feedback will be gathered through focus groups and interviews with an emphasis on opinions and use of current energy feedback along with what users would expect and want to see from improved feedback. As the visualisations are formulated based on early user feedback, subsequent focus groups will be able to discuss and respond to different designs of the visualisations. Once functional prototypes AR and VR applications using these visualisations are developed, they will be tested directly with new users to not only assess usability and engagement but to evaluate the effectiveness of the visualisations compared to IHDs and dashboard-based presentations of the same information. Due to the iterative nature of UCD, focus groups and testing will continue in tandem as necessary to ensure enough feedback is gathered and to give different potential users chance to respond to the applications and visualisations.

An overview of the UCD cycle employed for this research is shown in Fig. 2, which illustrates the iterative and progressive development stages based upon feedback from the different consumer groups. Each section has an accompanying

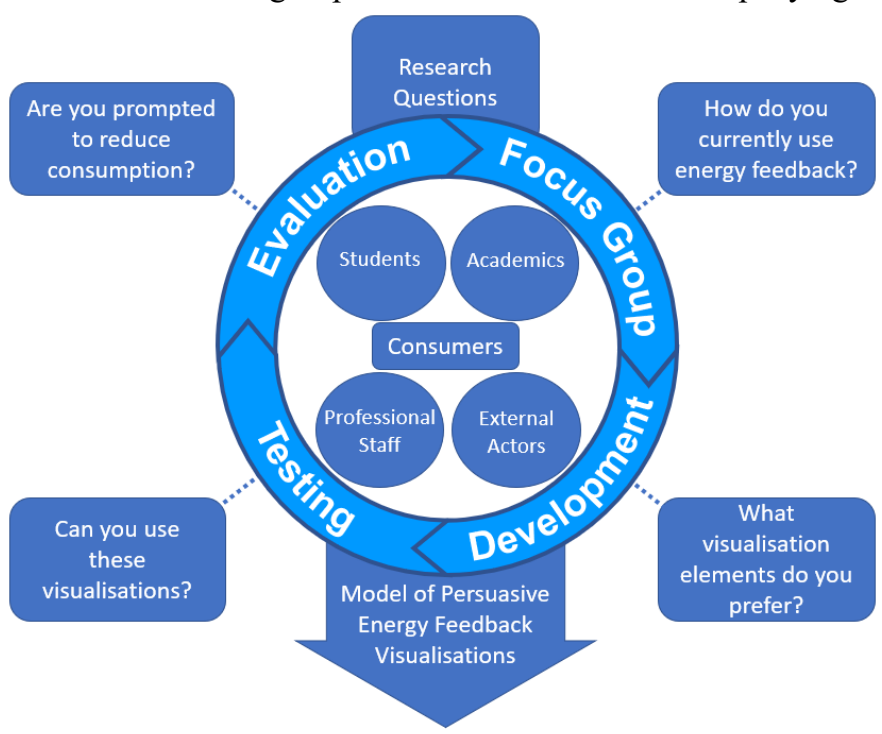

Figure 2. Overview of the User-Centred Design cycle employed for this research. overarching question that highlights the focus of the feedback at each stage of development. The feedback, testing and visualisation development will be used to guide a model of what visual elements are effective as persuasive visualisations for reducing consumption in energy feedback.

As this research is part of the SEND (Smart Energy Network Demonstrator) project, there are different potential user groups available for involvement in this UCD method. These groups are likely to provide different feedback and have different experiences and opinions of energy feedback in general.

\section{B. Smart Energy Network Demonstrator Project}

This research is being conducted as part of the SEND project at Keele University. The project will oversee the conversion of Keele University campus into a large-scale smart grid demonstration, highlighting new technologies and techniques related to smart energy research. This involves the installation of a multitude of new smart meters to gather energy consumption from across the campus. Due to this project encompassing the entire University, it will facilitate gathering participants from different groups throughout Keele, including students, academics, professional staff and external actors. Conducting focus groups from within each of these consumer groups will highlight the similarities and differences in how different types of consumers treat and respond to energy feedback.

As the SEND project is focused on smart energy at Keele University, the visualisations for this research will focus on the display of energy consumption data for the campus buildings. This presents several unique opportunities for the development of these visualisations. Ideally, the persuasive elements of the feedback driven visualisations will be usable both at institutes and in personal residences to promote energy conserving behaviours. Demonstrating the campus energy consumption visually however may help with some of the problems with home consumer energy feedback from the current literature. Consumers suggested an unwillingness to reduce consumption at times due to some actions only resulting in a miniscule saving as well as a feeling of abandonment by the government, companies and institutes [16]. Visualising the campus energy consumption will allow for demonstrations of how many consumers reducing their usage by a small amount can have an impact on overall consumption while providing a message for consumers that they are not alone in trying to reduce their resource use.

\section{IMPLEMENTATION}

Final implementation will depend on feedback gathered from users during the UCD process. For early testing, demonstrating and feedback reasons, the initial builds will be built using JavaScript and utilising standard Web Technologies. This means the visualisations are platform agnostic and can be used on most modern devices freely, but the performance and features will be more limited. To provide a variety of builds for users to feedback on, the initial builds will make use of the JavaScript libraries ar.js, tensorflow.js and three.js.

A marker-based AR visualisation will function with the use of ar.js, allowing easy demonstration of 3D models of the campus buildings and relevant models to result in a persuasive display. A building recognition and object segmentation AR build will utilise the deep learning tensorflow.js library. The 
built in HTML5 Canvas element can be used to superimpose effects straight on to the relevant buildings. For the initial VR build, three.js will allow a more in-depth $3 \mathrm{D}$ visualisation of the campus, where users can explore the displays of energy data for all the buildings from a single location.

The energy consumption data, including relevant information from other sources (such as building occupancy) will be sample data based on historical records for the early implementations of the research. As the SEND project and visualisations develop, the visualisations can start to make use of actual live data.

\section{CONCLUSIONS AND FUTURE WORK}

This research, as part of the SEND project, presents an opportunity to develop novel energy data visualisations based on feedback from different user groups in an institutional setting. These visualisations will use Augmented Reality and Virtual Reality as Persuasive Technologies to promote energy saving behaviours as current energy feedback, employed by Smart Meters and In-Home Displays, does not appear to be resulting in energy conservation behaviours. Visualising energy consumption at an institutional level provides an opportunity to develop a model for creating persuasive energy visualisations that can be applied to the domestic setting as well as providing support for individual consumer's concerns where energy feedback is lacking.

This paper has acted as a position paper, outlining the literature justification for this research and highlighting the methodology and process the subsequent research will follow. Future work will: Collate the opinions and responses from the different consumer focus groups, comparing between the different groups and against the current literature on energy feedback. Outline the feedback-driven visualisation development and highlight which stylised visualisation aspects work with consumers. And evaluate the effectiveness of such visualizations as persuasive technologies.

\section{ACKNOWLEDGMENT}

The Smart Energy Network Demonstrator project (ref 32R16P00706) is part-funded through the European Regional Development Fund (ERDF) as part of the England 2014 to 2020 European Structural and Investment Funds (ESIF) Growth Programme. It is also part-funded by the Department for Business, Energy and Industrial Strategy (BEIS). This research is undertaken in conjunction with Connexica, a Staffordshire based data analytics and visualisation solution company.

\section{REFERENCES}

[1] BEIS, 'Digest of UK Energy Statistics (DUKES) 2018: main report', Department for Business, Energy \& Industrial Strategy, Jul. 2018.

[2] BEIS, 'Provisional UK greenhouse gas emissions national statistics 2017', Department for Business, Energy \& Industrial Strategy, Mar. 2018.

[3] Masuma Ahmed, 'Smart Meters Quarterly Report to end June 2018 Great Britain Statistical Release: Experimental National Statistics', Department for Business, Energy \& Industrial Strategy, Aug. 2018.

[4] BEIS, 'Smart Meters Quarterly Report to end June 2018', Department for Business, Energy \& Industrial Strategy, Aug. 2018.

[5] J. Hamari, J. Koivisto, and T. Pakkanen, 'Do Persuasive Technologies Persuade? - A Review of Empirical Studies', presented at the Lecture Notes in Computer Science, 2014, vol. 8462, pp. 118-136.
[6] S. Darby, 'THE EFFECTIVENESS OF FEEDBACK ON ENERGY CONSUMPTION', Environmental Change Institute University of Oxford, Literature Review.

[7] K. Ehrhardt-Martinez, 'A Comparison of Feedback-Induced Behaviors from Monthly Energy Reports, Online Feedback, and In-home Displays', p. 15.

[8] J. Stromback, C. Dromacque, and M. H. Yassin, 'The potential of smart meter enabled programs to increase energy and systems efficiency: a mass pilot comparison Short name: Empower Demand', p. 92.

[9] M. A. Delmas, M. Fischlein, and O. I. Asensio, 'Information strategies and energy conservation behavior: A meta-analysis of experimental studies from 1975 to 2012', Energy Policy, vol. 61, pp. 729-739, Oct. 2013.

[10] J. Schleich, C. Faure, and M. Klobasa, 'Persistence of the effects of providing feedback alongside smart metering devices on household electricity demand', Energy Policy, vol. 107, pp. 225-233, Aug. 2017.

[11] D. Wemyss, F. Cellina, E. Lobsiger-Kägi, V. de Luca, and R. Castri, 'Does it last? Long-term impacts of an app-based behavior change intervention on household electricity savings in Switzerland', Energy Research \& Social Science, vol. 47, pp. 16-27, Jan. 2019.

[12] Lesic, V., Bruin, W. B. d., Davis, M. C., Krishnamurti, T. \& Azevedo, I. M. L. (2018), 'Consumers perceptions of energy use and energy savings: A literature review', Environmental Research Letters 13(3).

[13] T. Krishnamurti, A. L. Davis, G. Wong-Parodi, J. Wang, and C. Canfield, 'Creating an in-home display: Experimental evidence and guidelines for design', Applied Energy, vol. 108, pp. 448-458, Aug. 2013.

[14] Buchanan, K., Russo, R. \& Anderson, B. (2014), 'Feeding back about eco-feedback: How do consumers use and respond to energy monitors?', Energy Policy 73, 138-146.

[15] Schwartz, T., Stevens, G., Jakobi, T., Denef, S., Ramirez, L., Wulf, V. \& Randall, D. (2015), 'What People Do with Consumption Feedback: A Long-Term Living Lab Study of a Home Energy Management System', Interacting with Computers 27(6), 551-576.

[16] T. Hargreaves, M. Nye, and J. Burgess, 'Keeping energy visible? Exploring how householders interact with feedback from smart energy monitors in the longer term', Energy Policy, vol. 52, pp. 126-134, Jan. 2013.

[17] Fischer, C. (2008), 'Feedback on household electricity consumption: a tool for saving energy?', Energy Efficiency, 1(1), 79-104. 01206.

[18] Pahl, S., Goodhew, J., Boomsma, C. \& Sheppard, S. R. J. (2016), 'The Role of Energy Visualization in Addressing Energy Use: Insights from the eViz Project', Frontiers in Psychology 7.

[19] L. J. Becker and C. Seligman, 'Reducing Air Conditioning Waste by Signalling it is Cool Outside', Pers Soc Psychol Bull, vol. 4, no. 3, pp. 412-415, Jul. 1978.

[20] F. Quintal, C. Jorge, V. Nisi, and N. Nunes, 'What-I-See: A Tangible Visualization of Energy', in Proceedings of the International Working Conference on Advanced Visual Interfaces - AVI '16, Bari, Italy, 2016, pp. 120-127.

[21] W. G. Santika and I. K. G. Sudiartha, 'Persuasive technology with normative feedback to reduce energy consumption', in 2016 International Conference on Progress in Informatics and Computing (PIC), 2016, pp. 317-320.

[22] T. Kim, H. Hong, and B. Magerko, 'Design requirements for ambient display that supports sustainable lifestyle', presented at the DIS 2010 Proceedings of the 8th ACM Conference on Designing Interactive Systems, 2010, pp. 103-112.

[23] Olshannikova, E., Ometov, A., Koucheryavy, Y. \& Olsson, T. (2015), 'Visualizing Big Data with augmented and virtual reality: challenges and research agenda', Journal of Big Data 2(1), 22.

[24] Li, W., Nee, A. \& Ong, S. (2017), 'A State-of-the-Art Review of Augmented Reality in Engineering Analysis and Simulation', Multimodal Technologies and Interaction 1(3), 17.

[25] Garca-Hernndez, R. J., Anthes, C., Wiedemann, M. \& Kranzlmller, D. (2016), Perspectives for using virtual reality to extend visual data mining in information visualization, in '2016 IEEE Aerospace Conference', pp. 1-11.

[26] Patti, E., Mollame, A., Erba, D., Dalmasso, D., Osello, A., Macii, E. \& Acquaviva, A. (2017), 'Combining Building Information Modelling and Ambient Data in Interactive Virtual and Augmented Reality s 